\title{
Community Accessibility: Tweeters Take Responsibility for an Accessible Web 2.0
}

\author{
Kate Ellis, Mike Kent
}

\section{Introduction}

In his book on new forms of personal expression and communication on the Internet, The Future of Reputation, Daniel Solove reminds us that "people scattered across the globe can now all congregate together in cyberspace" (2007:33). He observes that social networking sites where people create profiles and alert the world of their thoughts and activities have emerged in an era where the idea of a local community in which everyone knows everyone is evolving into an atmosphere of individualism and of being alone in the global world. Twitter (www.twitter.com) in particular has been credited with making local events global. Martin Cahill (2009) described 2009 as "the year of 'Twitter". Established in 2006, the micro-blogging site is currently the third most popular social networking platform (Johnson 2009). Twitter was growing at a rate of 2,565 percent in early 2009 (Ostrow 2009a) and currently has more than 50 million user accounts (Pepitone 2010). These users generate approximately 1.5 billion tweets a month (Schonfeld 2010). In the United States at the end of 2009 19\% of all Internet users were using Twitter or a similar status update service (Fox, Smith and Zickuhr 2009). The 140 character limit on "tweets" or text-based posts was conceived to be compatible with SMS messaging and slang. For Steven Johnson (2009), Twitter's "remarkably simple" interface has spawned an exciting era of innovation where "the most fascinating thing about Twitter is not what it's doing to us. It's what we're doing to it'”

Our aim here is to use Twitter to engage with this new global-local village to explore the intersecting areas of critical disability studies, political mobilization and online social networking. The user created Twitter platform "Accessible Twitter" will be used as a case study to explore the importance of innovation and accessibility in Web 2.0. Accessible Twitter is accessible to users with a disability and vision impairment, in particular. Accessible Twitter is an important example of the kind of exciting innovations inspired by user-generated social networking and signals towards the future possibilities for an accessible Web. The first part of the paper focuses on the Web as a platform to connect people, locating the centrality of people with disability for Tim Berners-Lee when he conceived of the Web as a way to connect to each other. The paper then moves to briefly consider the political significance of Twitter as a platform for democracy and political activism using the 2009 uprisings in Iran in order to make a connection with people using Twitter to advocate for an accessible Web. We then introduce the idea of community accessibility where members of a network contribute to it to ensure it is accessible for other members. The final section of the paper situates Accessible Twitter within the continuum of Community Accessibility and political activism available via Web 2.0.

\section{The Power of the Web Is In its Universality}

In articulating his vision for a platform to share information, Tim Berners-Lee believed that access for everyone regardless of disability was a crucial factor: 
As we move towards a highly connected world, it is critical that the Web be useable by anyone, regardless of individual capabilities and disabilities...The W3C is committed to removing accessibility barriers for all people with disabilitiesincluding the deaf, blind, physically challenged, and cognitively or visually impaired. We plan to work aggressively with government, industry and community leaders to establish and attain Web accessibility goals. (Goggin and Newell 2003:109)

However, the increasing use of graphics, user generated content and a rejection of Web standards, is blocking out people with disability (Craven 2008). As Gerard Goggin and Christopher Newell (2003) argued in their seminal book on this topic Digital Disability the Internet will not be fully accessible until disability is considered a cultural identity in the same way that class, gender and sexuality are. For Goggin and Newell (2003) digital technologies, including the Internet, broadband and advanced telecommunications have the potential to revolutionize our lives and have been held up as a way to eradicate disability yet disappointingly continue to "build in" disability at the point of production. Since the time of their writing in 2003, there have been massive shifts in the way the Web is used and developed. Accessibility, which is more widely understood, is ignored due to a continuation of the social prejudice that Goggin and Newell sought to expose. Following the publication of their text, the Web has evolved to Web 2.0 and has generally become less accessible. This paper builds on their ideas and applies them to Twitter and social networking (a more recent Web 2.0 phenomenon). Chieko Asakawa, a vision impaired researcher, has published widely on the non-visual Web and sees an enormous accessibility chasm between the early Internet and the more recent Web browsing experience:

Lynx, a text-based Web browser, which was developed in 1992, was very accessible for screen reader users, since it could be controlled with just a keyboard. By pressing the curser right or left keys, for example, users could move to the next or previous link texts. I was able to access the Web for the first time using linux in 1994, with a DOS screen reader through telnet by accessing a UNIX server. (Asakawa 2005)

She argues that the plugins and multimedia which characterize Web 2.0 prevent blind Web users from authentically experience the Web. Of course the ability of most people to access the World Wide Web in 1994, regardless of any level of disability, was quite limited, with not everyone possessing Asakawa's considerable skills and literacies in this, at the time, very new technological medium. This ironically also meant that this lack of access was less of a disabling factor at the time. The first widely distributed Web browser Mosaic had only been launched in 1993 and in 1994 there were less than 50 million Internet users, mostly concentrated in North America. By contrast in late 2009 there were more than 1.7 billion Internet users. The Internet has gone from a relatively marginal medium in the early to mid 1990s to one that is much more mainstream in 2010; ironically, this growth in the use of the medium has also tracked with it becoming less accessible for people with disabilities.

\section{| Connecting People}

Twitter combines the concept of social networking with SMS communication to rework the values of local community within a global environment:

In countries all around the world, people follow the sources most relevant to them and access information via Twitter as it happens - from breaking world news to updates from friends. (Twitter n.d.)

This quote from the "About us" section of Twitter highlights the global nature of social networking and its importance in keeping the average world citizen up to date with what's happening in their relevant networks whether they be local or global. Social networking sites, such as Facebook, MySpace and Twitter, encourage an increased level of documentation so although ties may be weak, the possibility of obscurity is low. Further, this is happening in a worldwide sense. Critics of this social environment suggest mobile phone directories and instant messaging are a poor replacement for "true networks of kinship" (Bauman 2004:130). The Internet and mobile access are reinventing aspects of the local village in a global environment. Twitter, in particular, has been embraced by people using mobile devices to access the Internet as a way of maintaining contact with their extended social network (Fox et al. 2009). Twitter is developing differently to previous social networks; rather than being embraced by young early adaptors, Twitter has been described as 'aging in reverse' (Ostrow 2009b) as it was initially embraced by older users and is only more recently being embraced by a younger demographic. The median age of Twitter users is still 31 
although presumably falling compared to Facebook's 26 and MySpace's 27. This older demographic and the public embrace of the platform by people in the news media, celebrities and politicians has lead to the impression that Twitter punches above its weight in terms of influence in the social media marketplace, particularly when compared to more established names such as Facebook and MySpace. When Tim Berners-Lee articulated his vision for the World Wide Web he focused on connecting people, including people with disability and those from other countries and cultures. When he launched the International Program Office for Web Accessibility Initiative he emphasized the importance of "operation across different hardware and software platforms, media, cultures and countries" (Berners-Lee 1997). The Web has undoubtedly benefitted people with disability in their work, home and leisure pursuits. However, amongst this celebration of increased opportunity we must take care not to gloss over issues of accessibility. Becky Gibson (2007) cites user generated content as one particularly troublesome area:

The next generation of the Web is relying on new technologies to build rich interfaces and applications which enable community, collaboration, social networking and enhanced interactions. This has implications for people with disabilities who have come to rely on the Web to provide more independence, work opportunities, and social interactions. (P. 2)

User generated content is providing challenges for universal design and disability. Accessibility features in protocols such as Flash animations, for example, are unknown to most users and hence provide limited potential for those who require different forms of access. Digital media and online technology hold the promise that people with disability will be included; however, features that enhance accessibility for groups with a certain type of impairment can diminish accessibility for another. Just as people don't wish to be locked into a particular method of generating content, people should not be locked into a single method of viewing content. This focus on "disability" potentially crowds the focus on the ways in which technologies can be redesigned to eliminate the ablest oppression that keeps people with disabilities from being able to participate fully in all aspects of the material and social world. The importance of establishing new technologies to address accessibility must be put into practice. Accessibility must be built into the initial toolkit and flow on to end users because the creation of a blog, wiki or Web page does not involve complex technical knowledge or skills (Gibson 2007).

\section{| Political Significance}

Twitter claims a mix of public and private technologies that allow an easier global connection. It is predominately a social networking system and is heavily dependent on the mobile Web. In this way, personal moments can be transformed into wider social and political issues as Giroux (2009) suggests of the broadcast of the democratic protests in Iran in June 2009. For Giroux, new communication technologies and especially social networking sites are far more than depoliticized "personal tools" or "entertainment devices" (2009). A combination of social networking and mobile technology awakened a "reservoir of political activity" allowing people within and outside Iran access to information that would have otherwise been stifled (Giroux 2009). 140 character "tweets" and photo links were an effective method to disseminate information following the limitations placed on mainstream media during the conflicts. Political activists in China have likewise embraced the microblogging site and access was blocked by the Chinese government to prevent discussion of the 20th anniversary of the Tiananmen Square massacre. Twitter has also been used recently in both Egypt and Moldova (Giroux 2009). Although our position regarding Twitter's role in political mobilization is celebratory, others are more cautious suggesting that the only reason Twitter was not blocked in Iran was because the government used it for surveillance (Lake 2009). While Twitter may not be inherently political, its format has invited political mobilization in many areas, including disability and accessibility. Twitter has attracted a number of people interested in promoting the importance of Web accessibility, including the W3C Web Accessibility Initiative (WAI) - who have a Twitter account. The strong accessibility community on Twitter is giving a public exposure to issues related to disability that have historically been considered personal problems that an individual must overcome. As we write this, some accessibility tweets on Twitter include:

really when will ppl get accessible site $=$ mobile site $!$ :) dropdown is basically unusable with mobile browser

hearing some very early, but very encouraging rumors about possible future platforms for W3C Widgets ... exciting!

I had a look on YouTube last night at a video for the accessibility features, and I was very impressed! Mum liked them too :D 
Not \#accessibility, but \#UniversalDesign. What is good for PWD is good for business. Reduce web dependencies \&amp; open markets. \#ally

\section{Community Accessibility}

Marlee Matlin, perhaps the most famous Deaf Hollywood actress, recently used her Twitter account to launch an attack on the lack of captioning of online videos:

20 years ago I fought the fight for captions. And the law was passed 17 yrs ago. Now I'm forced to begin again. I'm screaming NOW not later.

The online realm which is being revolutionized by network television is exempt from the requirement to provide captions for shows aired on television. While Matlin is focusing on the corporate leaders such as iTunes that make money out of this and have revolutionized television viewing, Berners-Lee has been questioned by video bloggers as to whether they need to provide captions. He acknowledges the difficulties with this and suggests "community captioning" in the spirit of Web 2.0 where "The video blogger posts his blog - and the Web community provides the captions that help others" (OUT-LAW News 2006). This solution embraces the collaboration that underscores Web 2.0 and highlights the importance of Apple and other online network video services to lead the way in this area. While there are a number of people who use Twitter to tweet about accessibility issues, Twitter itself in inaccessible. Dennis Lembree outlines several inaccessible features of Twitter:

- No keyboard access to favorite/reply/delete

- Lack of headings

- Favoriting requires Javascript

- Custom colors may not be readable

- Code doesn't validate

- Code could use better semantics

- $\quad$ Password field is missing a label; select lang missing label

- Fieldsets used without legends

- Layout width is static not flexible; doesn't "stretch" with browser width

- JavaScript required for details on latest tweet (time, in reply to)

(DeNardis 2009)

Given that Twitter is primarily a text-based medium, many of their accessibility problems could be easily solved. Cahill (2009) believes that Twitter, which currently has no accessibility policy, needs to start addressing accessibility in order to reach the number one social networking spot because if a site is "accessible, easy to navigate and read, it is typically successful". Solving these accessibility issues would also likely make it the most accessible social networking site: a significant competitive edge given that Facebook recently overhauled its accessibility features. Lembree argues that people with vision impairment are being excluded from Twitter and the Web, in general, because of a focus on more complicated programming and a desire to save money. Although he believes people with disability are "not intentionally neglected online", inaccessibility is a determining point of the way we interact with social networking technology and reflects the disabling values of the people who design and maintain these limiting applications (Foley and Voithofer 2008). The assumption that every Web user has the same ability to access the Web is an intentional example of "doing production." (Goggin and Newell 2003). The production of disability as a category is a dynamic process that is shaped by technology, culture and the underpinning moral order; disability only exists in society (Goggin and Newell 2003:21). Disability is a negative social interpretation of impairment that empowers dominant groups and institutions. A critical understanding of disability in line with other marginalized groups will enable a realization of Tim Berners-Lee's vision for an entirely accessible connected Web because accessibility will be prioritized. Alan Roulstone suggests that new technology is experienced according to the conditions of the existing social and theoretical order. Technology remains influenced by existing social values (Roulstone 2010:7). 


\section{Accessible Twitter}

Twitter's simple design invites collaboration amongst the Twitter interface, users and third party platforms. The community is encouraged to improve the service and the more people that use it the better it gets; in this way, "users add value". In the spirit of Web 2.0 users are treated as "co-developers" (O'Reilly 2005) with amateur codes allowing users to organize their feeds, upload videos and locate other users within a certain distance. Similarly, Accessible Twitter, allows more people to join the network and was developed by a small startup. Dennis Lembree won the 2009 Access IT@web2.0 Award for his Twitter tool “Accessible Twitter”. Lembree, whose speciality in Web development is standards and accessibility overhauled the Twitter site to make it accessible to users who are blind or vision impaired. Although motivated by disability and accessibility, Lembree argues that the benefits of Web accessibility reach far beyond this group as well. Lembree has addressed many of the inaccessible features of Twitter to ensure it adheres to Web standards. Lembree was unable to maintain the traditional Twitter "look" because it does not lend well to accessibility; so, Accessible Twitter has a different "look" to Twitter. Accessible Twitter adopts a simple and consistent layout, and navigation can work with or without JavaScript. JavaScript is a functional programing language that is used to make Web pages more dynamic and interactive. As an add-on to html it has traditionally posed a problem for users with disability. In the first set of Web Standards WCAG1.0, it was a top priority that Web pages work without javascript; however, more recently screen readers have developed the capability to handle it and it is not highlighted as an issue in WCAG 2.0 at all. Designed with Web 2.0 technology in mind, WCAG 2.0 encourages flexibility and requires that all Web sites be perceivable, operable, understandable and robust. Nevertheless as a proportion of people continue to browse without JavaScript and many people with vision impairment use older versions of screen reading software; it is still an important business and accessibility issue to ensure the page works with or without JavaScript. Lembree has also ensured "forms are marked up for optimal accessibility". This is a relatively easy to achieve feature which includes clear identification and labeling as well as allowing exclusive use by both a keyboard and mouse. This is an important feature for users with a mobility or vision impairment who can't use a mouse and people without disability who prefer to use keyboard shortcuts as a time saver. Accessible Twitter has a large default text size that adopts a high color contrast making it perceivable and understandable. The site allows users to access it in either high or low resolution in line with Berners-Lee's original vision for the Web. Finally, Lembree has made a commitment to expanding the accessibility features of Accessible Twitter "every day". As we write this Accessible Twitter announced an app update:

App Update: due to API inconsistency, tweets on user profile page reduced from 20 to 1; added a link to view last 20 \#api

Lembree was not surprised at the inaccessibility of the original Twitter even given its reputation for being simple and easy to use because of the misconception that a site can't be cool and accessible at the same time. For Lembree, ensuring functionality and fun together is a simple matter of "planning it from the beginning and implementing progressive enhancement". Lembree's Accessible Twitter has been enthusiastically received by those with and without vision impairment in the Twitterverse. The project has raised the visibility of the importance of accessibility in the rapidly evolving online world:

Wow! You have really made my day, and I am smiling once again. I am so excited that I found your Accessible Twitter. Now I feel that I can be in the cutting edge of everything that is happening in the Twitter Universe.

$$
\text { from Jacqueline (via Dennis' blog) }
$$

Many thanks to @accessibleTwitr for his very nice Twitter Web application!

$$
\text { from PerBusc }
$$

Lembree claims that the inaccessibility of Twitter is a general reflection of the poor quality of accessibility on the Web:

Twitter.com is extremely off-course as far as web accessibility goes. Basic things such as proper use of headings and keyboard access are not implemented. I received an email from a blind user who really enjoys Accessible Twitter. He even asked, I think jokingly, if I could make an accessible Facebook, which is also terribly inaccessible. (Accessify 2009)

Although Twitter did not even rate a mention, in 2008 AbilityNet found a number of social networking sites 
were inaccessible to users with a number of different disabilities. Since that time the importance of accessibility has been demonstrated with Facebook and YouTube, two sites criticized by AbilityNet, revamping their accessibility features with far reaching success in audience satisfaction while MySpace, which was also criticized has done nothing and lost 54\% of its market share (Cahill 2009). Cahill believes Twitter should take notice and the existence of Accessible Twitter does not let them off the hook:

Accessible Twitter is designed to be easier to use and is optimized for disabled users, but it is yet to be seen whether such a move will prompt Twitter into action or have the reverse effect, letting them feel that they can more easily shed all responsibility. In such a competitive market and with other social media players innovating and leading on accessible design, accessible design is becoming central to the development and continued growth of online businesses. This fact should be food for thought for a savvy startup like Twitter. Leaving it too long might just signal the beginning of the end.

(Cahill 2009)

\section{Conclusion}

Clearly, Web 2.0 accessibility is of most benefit to people with disabilities and especially those with vision impairments, older people and people in the developing world, the recent trend toward mobile applications and Internet usage suggests the majority of Internet users would benefit from accessibility options (Zajicek 2007:1). The move from "Reading the Web" to "Writing the Web" is an important point in the evolution of the Internet in cementing its place in our lives. Although a plethora of avenues exist in terms of generating content, opportunities for how this information is accessed and viewed is limited to the restrictions established by the creators of sites. In theory, digital information can be accessed by users with different needs in different ways as Asakawa's early experiences demonstrate. The user can determine the way the information is displayed visually, the size of the text and its background, or alternately have images relayed as sounds through a screen reader, or as refreshable Braille using a Braille output device. However, in practice this is not always the case, particularly as the Web becomes more complex and a more ubiquitous part of life,Despite its reputation for being simple and easy to use and its perceived importance as a tool for democracy and political mobilization, Twitter builds in disability at the point of production by failing to consider accessibility. However, at the same time by encouraging a community of members to create Twitter applications, Berners-Lee's belief that accessibility can be achieved via a network of community is realized. Lembree's Accessible Twitter shows that accessibility in social networking is possible. While Lembree's adoption of community accessibility gives cause for great optimism, it is equally important for the corporations to lead the way by building accessibility features into their toolkits.

\section{References}

Accessify. 2009. "Interview with Accessible Twitter Creator Dennis Lembree." April 23. Retrieved March 29, 2010 (http://accessify.com/news/2009/04/ interview-with-accessible-twitter-creator-dennis-1-embree/).

Asakawa, Chieko. 2005. "What's the Web Like If You Can't See It?” ACM International Conference Proceedings Series: Vol 88. Chiba, Japan: Proceedings of the 2005 International CrossDisciplinary Workshop on Web Accessibility (W4A). Retrieved March 29, 2010 (http://www.ra.ethz.ch/CDstore/www2005ws/workshop/wf01/1-Asakawa.pdf).

Bauman, Zygmunt. 2004. Wasted Lives. London: Polity Press.
Berners-Lee. 1997. "World Wide Web Consortium Launches International Program Office for Web Accessibility Initiative." October 22. Retrieved March 29, 2010 (http://www.w3.org/ Press/IPO-announce).

Cahill, Martin. 2009. "A Wake-Up Call For Twitter." NewMatilda.com. December 1. Retrieved March 29, 2010 (http://newmatilda.com/2009/12/01/wakeup-call-twitter).

Craven, Jenny. 2008. "Web Accessibility: What We Have Achieved and Challenges Ahead.” The World Library and Information Congress. Quebec, Canada, August 10-14. Retrieved March 29, 2010 (http://archive.ifla.org/IV/ifla74/ papers/086-Craven-en.pdf). 
DeNardis, Nick. 2009. "Accessible Twitter." .eduGuru February 16. Retrieved March 29, 2010 (http://doteduguru.com/ id2215-accessible-twitter.html).

Foley, Alan and Rick Voithofer. 2008. "Social Networking Technology, NetGen Learners, and Emerging Technology: Democratic Claims and the Mythology of Equality." Presented at the American Educational Research Association (AERA) National Conference, New York, New York: March 24-28. Retrieved March 29, 2010 (http://Alynnfoley.typepad.com/ FoleyVoithofer.pdf).

Fox, Susannah, Aaron Smith, and Kathryn Zickuhr. 2009. “Twitter and Status Updating, Fall 2009.” Pew Internet and American Life Project. October. Retrieved March 29, 2010 (http://www. pewinternet.org/Reports/2009/Twitter-and-status-updating. aspx).

Gibson, Becky. 2007. "Enabling An Accessible Web 2.0." ACM International Conference Proceeding Series Vol. 225. Proceedings of the 2007 International Cross-disciplinary Conference on Web accessibility (W4A). Banff, Canada, May 7-8. March 29, 2010 (http://www.w4a.info/2007/prog/ k1-gibson.pdf).

Giroux, Henry. 2009. "The Iranian Uprisings and the Challenge of the New Media: Rethinking the Politics of Representation." Fast Capitalism 5.2. Retrieved March 29, 2010 (http://www. uta.edu/huma/agger/fastcapitalism/5_2/Giroux5_2.html).

Goggin, Gerard and Christopher Newell. 2003. Digital Disability: The Social Construction of disability in New Media. Oxford: Rowman and Littlefield.

Johnson, Steven. 2009. "How Twitter Will Change the Way We Live." Time in partnership with CNN. June 5. Retrieved March 29, 2010 (http://www.time.com/time/ printout/0,8816,1902604,00.html).

Lake, Eli. 2009. "Iran Prepared to Track Dissent on Social Networks." The Washington Time. June 20. Retrieved March 29, 2010 (http://www.washingtontimes.com/news/2009/ jun/20/iran-has-tech-to-track-tweets-texts/).

O’Reilly, Tim . 2005. "What is Web 2.0: Design Patterns and Business Models for the Next Generation of Software." O’Reikky. September 30. Retrieved March 29, 2010 (http:// oreilly.com/web2/archive/what-is-web-20.html).
Ostrow, Adam. 2009a. "The Fastest Growing Social Sites." Mashable: The Social Media Guide April 20. Retrieved March 29, 2010 (http://mashable.com/2009/04/20/ the-fastest-growing-social-sites/).

---. 2009b. "STATS: Younge People Are Flocking to Twitter." Mashable: The Social Media Guide September 2. Retrieved March 29, 2010 (http://mashable.com/2009/09/02/twitter-demographics/ ).

OUT-LAW News. 2006. "Berners-Lee Applies Web 2.0 to Improve Accessibility." May 24. Retrieved March 29, 2010 (http://www.out-law.com/page-6946).

Pepitone, Julia. 2010. “Twitter Users Not So Social After All." CNNMoney.com. March 10. Retrieved March 29, 2010 (http://money.cnn.com/2010/03/10/technology/twitter_ users_active/index.htm?section=yahoo_buzz). Roulstone, Alan. 2010. Enabling Technology: Disabled People, Work and New Technologies. New York: Routledge.

Schonfeld, Erick . 2010. "Twitter Hits 50 Million Tweets Per Day.” Tech Crunch February 22. Retrieved March 29, 2010 (http://techcrunch.com/2010/02/22/twitter-50-milliontweets-day/).

Solove, Daniel. 2007. The Future of Reputation: Gossip, Rumour, and Privacy on the Internet. New Haven: Yale University Press.

Twitter. 'About Us' (n.d.). Retrieved March 29, 2010 (http:// twitter.com/about\#about).

Zajicek, Mary. 2007. "Web 2.0: Hype or Happiness?" Proceedings of the 2007 International Cross-disciplinary Conference on Web accessibility. Banff, Canada: May 7-8. Retrieved March 29, 2010 (http://www.google. com.au/url sa $=t \&$ source $=$ web $\& c t=r e s \& c d=1 \& v e d=-$ 0CAYQFjAA\&url=http\%3A\%2F\%2Fciteseerx.ist.psu. edu\%2Fviewdoc\%2Fdownload\%3Fdoi\%3D 10.1.1.108.63 90\%26rep\%3Drep 1\%26type\%3Dpdf\&ei=EciwS5qGHJC-rAeC8PmmAQ\&usg=AFQjCNH0uq2YxTEIt8Std1Jn amVNluM7Q). 
